\title{
Accelerating Universes from Compactification on a Warped Conifold
}

\author{
Ishwaree P. Neupane* \\ Department of Physics and Astronomy, University of Canterbury, Private Bag 4800, Christchurch 8020, New Zealand \\ and Theory Division, CERN, CH-1211 Geneva 23, Switzerland
}

(Received 26 September 2006; revised manuscript received 2 January 2007; published 7 February 2007)

\begin{abstract}
We find a cosmological solution corresponding to the compactification of 10D supergravity on a warped conifold that easily circumvents the "no-go" theorem given for a warped or flux compactification, providing new perspectives for the study of supergravity or superstring theory in cosmological backgrounds. With fixed volume moduli of the internal space, the model can explain a physical Universe undergoing an accelerated expansion in the 4D Einstein frame, for a sufficiently long time. The solution found in the limit that the warp factor dependent on the radial coordinate $y$ is extremized (giving a constant warping) is smooth and it supports a flat four-dimensional Friedmann-Robertson-Walker cosmology undergoing a period of accelerated expansion with slowly rolling or stabilized volume moduli.
\end{abstract}

DOI: 10.1103/PhysRevLett.98.061301

Introduction.-Recent astronomical data, notably the observations of high redshift type Ia supernovae [1] and measurements of the cosmic microwave background [2], not only provide emerging evidence for the ongoing accelerated expansion of the Universe but also provide support for the concept of inflation, or a rapid exponential expansion of large magnitude in a much earlier cosmological epoch. Although it is not difficult to construct cosmological models that exhibit these features, one would prefer any such model to be derivable from a fundamental, and mathematically consistent microscopic theory of (de Sitter) quantum gravity, such as string theory. Superstring theory lives in ten dimensions but we live in a four-dimensional Universe. Clearly, any attempt to derive a viable cosmology from string or $M$ theory (compactification) must produce a four-dimensional de Sitter Universe similar to ours and the size of extra dimensions should remain much smaller than the physical three dimensions.

The past few years have witnessed significant progress in building of inflation models within string theory via flux compactifications of the ten- or eleven-dimensional spacetime of superstring or $M$ theory with the desire to find models for late-time cosmology [3-5] supporting a small positive cosmological constant. If one wishes to stay within the realm of low energy supergravity models derived from superstrings, cosmic inflation is ruled out for warped flux compactifications of classical supergravities on the basis of a "no-go" theorem [6,7], which forbids accelerating solutions for warped (and static) extra dimensions. For a way out, one may possibly include higher curvature corrections [8] to the leading order Lagrangian in $\alpha^{\prime}$ expansion or extended sources (branes, antibranes) that are present in string theory [9] or even invoke certain nonperturbative effects (such as, gaugino condensate and Euclidian D3 branes) [3]. These all achieve some limited success in overcoming the no-go theorem. However, there is no good reason to suppose that all these string effects are available at much lower energy scale, such as $\rho_{v \mathrm{ac}} \sim$ $10^{-3} \mathrm{eV}$ or $H \sim 10^{-60} M_{\mathrm{Pl}}$. There is another particular
PACS numbers: 98.80.Cq, 11.25.Mj, 11.25.Yb

difficulty in this program in stabilizing the common modulus associated with the overall shape and size of the internal Calabi-Yau spaces. Freezing the volume moduli using nonperturbative dynamics seems beyond anything visible in supergravity. Time and space are not independent, so any idea that the geometry of spacetime is fixed and nondynamical is probably wrong. The advent of string or $M$ theory in time-dependent backgrounds is an important and promising subject. It has the potential to offer a resolution to the dilemma posed by the observed cosmic acceleration within a natural theoretical framework. In [4] (and generalizations thereof [5]) the no-go theorem was circumvented just by the choice of negatively curved internal spaces, once the fluxes are turned off. The restriction on the curvature seems a severe one, especially given the view that flat space compactifications on Calabi-Yau spaces are among the most natural in string theory.

An interesting observation in [10] is that a timedependent compactification of classical supergravities with Ricci-flat extra spaces, involving certain twists in the geometry, can give rise to a positive potential in lower dimensions and hence a period of accelerated expansion in the 4D Einstein frame. It has been learned that the timevarying volume moduli with no initial fine tuning among the scalars lead only to a transient period of cosmic acceleration, except in the case that we live in a hyperbolic Universe [5]. To fully account for the nature of an effective four-dimensional cosmology, it is important to gain a proper description of spacetime dependent compactifications (of higher dimensional gravity), rather than the time-dependent or the space-dependent (warped) compactification alone. This is because upon dimensional reduction an internal space of positive curvature gives a negative potential in time-dependent backgrounds, while it gives a positive potential in standard warped backgrounds. In view of this observation, all the studies on flux and timedependent compactifications (of string or $M$ theory) to date are either incomplete, or are at best part of a more complete story. 
In this Letter we consider a particular model where the internal spaces have geometries specified by more scalars than just the volume modulus. The type IIB supergravity theory in ten dimensions, with a warped 6D conifold geometry provides an example of this kind, as originally studied by Klebanov and Tseytlin [11], and Klebanov and Strassler [12]. In this model, the internal space is a Ricciflat 6D cone $Y_{6}$ whose base is a 5D Einstein-Kähler space, $X_{5} \equiv T^{1,1}$. The introduction of branes may be important for constructing gauge field theories (of the elementary particles) at the tip of a warped conifold, given a view that both gravitational and nongravitational forces can be localized on D3 branes. We show that the "no-go" theorem does not apply to a time-dependent background even if the extra dimensions are warped.

The model. - We shall assume that ten- or elevendimensional supergravity is the relevant starting point. The model below corresponds to the dimensional reduction to four dimensions of type IIB supergravity, where the spacetime is a warped product of a six-dimensional space $Y_{6}$ and $M_{4}\left(\equiv \mathbb{R}^{1,3}\right)$. In particular, the $10 \mathrm{D}$ metric is

$$
d s_{10}^{2}=h^{-1 / 2} \tilde{g}_{\mu \nu} d x^{\mu} d x^{\nu}+h^{1 / 2} d s_{6}^{2} .
$$

The metric of the large three dimensions (plus time) is

$$
d x_{\mu} d x^{\mu}=-a^{2 \delta} d u^{2}+a^{2}\left(d x_{1}^{2}+d x_{2}^{2}+d x_{3}^{2}\right),
$$

where $a \equiv a(u)$ and $\delta$ is a constant, the choice of which fixes the nature of the time coordinate $u$. In the gauge $\delta=$ $0, u$ becomes the proper time $t$. The metric on the transverse $6 \mathrm{D}$ space is

$$
\begin{aligned}
d s_{6}^{2}= & e^{2 \alpha} g d y^{2}+e^{2 \beta} k \sum_{i=1}^{2}\left(d \theta_{i}^{2}+\sin ^{2} \theta_{i} d \phi_{i}^{2}\right) \\
& +e^{2 \sigma} m\left(d \psi+f \sum_{i=1}^{2} \cos \theta_{i} d \phi_{i}\right)^{2} .
\end{aligned}
$$

The ranges of the angular coordinates are $0 \leq \theta_{i}<\pi, 0 \leq$ $\phi_{i}<2 \pi$, and $0 \leq \psi<4 \pi$. We assume that the moduli parameters other than the volume scalars are stabilized (frozen); the scalars $\alpha, \beta$, and $\sigma$ are functions of $u$ (or the proper time $t$ ), while $g, k, m$, and $h$ are functions only of the radial coordinate $y$. We will also consider some examples where $\beta$ and $\sigma$ are functions of the radial coordinate $y$. As discussed in [12], due to the twist along the normal $S^{1}$ bundle, the model preserves only $\frac{1}{4}$ of the $\mathcal{N}=$ 4 supersymmetries and gives a mass to the scalar fields. Even though we put in different functions of $y$ for the two 2 -spheres and the twisted $S^{1}$, Einstein's equations simplify a lot when these functions are proportional, so henceforth $k(y) \propto m(y)$. The Einstein-frame metric $g_{E, \mu \nu}$ is related to $\tilde{g}_{\mu \nu}$ via

$$
g_{E, \mu \nu}=e^{2 \phi} \tilde{g}_{\mu \nu} .
$$

One must choose the scalars to satisfy $\alpha+4 \beta+\sigma \equiv 2 \phi$, so that the 4D Newton constant is then time independent; $\phi$ is a $4 \mathrm{D}$ scalar rather than the $10 \mathrm{D}$ dilaton.

The background solution. - The metric considered by KT [11] corresponds to the choice $f=1, \alpha=0, \beta=$ $-\ln \sqrt{6}, \sigma=-\ln 3, g=1$ and $k=y^{2}$. By turning on $N$ units of the NS 5-form flux on $X_{5}$ and $M$ units of the RR 3form flux through the $S^{3}$ of $T^{1,1}$, one finds [11]

$$
h(y)=h_{0}+\frac{L^{4}}{y^{4}}\left[1+\frac{3 g_{s} M^{2}}{8 \pi N}\left(1+4 \ln \frac{y}{y_{0}}\right)\right],
$$

with $L^{4} \equiv 27 \pi g_{s} N \alpha^{12} / 4$, which satisfies the standard quantization conditions: $\left(4 \pi^{2} \alpha^{\prime}\right)^{-2} \int_{T^{1,1}} F_{5}=N$ and $\left(4 \pi^{2} \alpha^{\prime}\right)^{-1} \int_{S^{3}} F_{3}=M$. The singularity at $y=0$ may be resolved by deforming the conifold $[12,13]$ or by allowing time dependence to the internal space.

Let us momentarily set $h \equiv$ const (or take $y \gg L$ ), $g \equiv$ 1 and $k \equiv y^{2}$, which is relevant to finding a pure timedependent solution. Einstein's equations admit the following explicit solution (in the gauge $\delta=3$ ):

$$
\begin{aligned}
& a=a_{0} e^{ \pm 2 c_{1} u}, \quad \alpha=c_{1} u+\alpha_{0}, \\
& \beta=c_{1} u+\alpha_{0}-\ln \sqrt{6}=\sigma+\frac{1}{2} \ln \frac{3 f^{2}}{2} .
\end{aligned}
$$

For the branch $c_{1} u<0$, the size of the internal space shrinks with time, while the size of the physical three spaces can grow if we choose the negative exponent. This result is remarkable as it was impossible for internal spaces with a single (common) volume modulus.

A few remarks may be relevant before we proceed. We have chosen a factorizable geometry: the dependence of the warp factor $h$ on time $t$ (or $u$ ) has been absorbed into the metric $\tilde{g}_{\mu \nu}$ (or the scalars $\alpha, \beta, \sigma$ ) as we would like to write the metric in 4D Einstein conformal frame; a time dependence in $h$ would render it difficult for such an interpretation. Time-dependent solutions of our sort were studied in the past, for example, by Kodama and Uzawa [14]. However, it was assumed there rather implicitly that $\alpha=\beta=\sigma$ and also $g=k=m$. In the work of Buchel, for example [15], the metric was not written in the 4D Einstein frame, and also no time dependence was allowed for internal spaces. These or other similar assumptions exhaust some (or all) of the interesting cosmological solutions that we have found in this Letter.

Kachru et al. [3] proposed to fix the volume moduli using some nonperturbative dynamics, such as a gaugino condensate. This is an interesting proposal but such a construction is model or scheme dependent. For the warped (conifold) geometry under consideration, the gaugino condensate is related to the deformation of the conifold, so it is already visible in the classical geometry, and one does not need instantons to see the condensate. However, we show that the volume moduli can be stabilized spontaneously due to a natural expansion of the Universe, even leading to a transient period of cosmic acceleration at late times. In 
general, the volume factors are dependent on both time and space; in order to write an effective action in four dimensions, it is necessary to integrate out the $y$ coordinate. This can be done only if the solutions for both space- and timedependent parts of the volume factors are known, simultaneously.

Cosmological solution. -We shall consider the case $\alpha=0$ and $g=1$, so as to maintain the interpretation of $y$ as the holographic energy scale. In the zero flux limit of the 10D Einstein equations, and with the choice $k \equiv y^{2}$, the symmetries of the metric ansatz imply that (i) $4 \beta+\sigma \equiv \varphi(t), \quad h(y)=\rho^{2} / y^{2}, \quad$ (ii) $4 \beta+\sigma=$ const $\equiv \mu, h(y)=\sqrt{\lambda+\rho^{4} / y^{4}}$. Upon dimensional reduction the first branch above yields $I=\frac{\operatorname{vol}\left(X_{5}\right)}{8 \pi G_{10}} \times$ $\int d^{4} x \int d y \sqrt{-g_{(4)}}\left(R_{(4)}+\mathcal{L}\right)$, where $\operatorname{vol}\left(X_{5}\right)$ contains only the space-dependent part,

$$
\begin{aligned}
\mathcal{L} \equiv & K-V=12 \dot{\beta}^{2}+\frac{3}{2} \dot{\sigma}^{2}+4 \dot{\beta} \dot{\sigma}-\frac{1}{2} \frac{h^{\prime 2}}{h^{3}} e^{-\sigma-4 \beta} \\
& -\frac{1}{y^{2} h}\left(f^{2} e^{\sigma-8 \beta}-4 e^{-\sigma-6 \beta}+20 e^{-\sigma-4 \beta}\right),
\end{aligned}
$$

where $^{\prime} \equiv d / d y$. The corresponding scalar potential allows only an anti-de Sitter minimum or it at best describes only a short period of accelerated expansion due to a relatively large slope of the potential along the $\beta$ direction. One can easily modify the form of the potential by introducing a bulk cosmological term or additional source terms (fluxes, branes) or even by invoking some particular nonperturbative dynamics, so as to uplift the anti-de Sitter minimum and make it a metastable de Sitter ground state. However, we do not consider this last possibility here, as it hinders our ability to find analytic solutions.

Instead we consider the second branch, (ii). The type IIB supergravity equations may be solved by making appropriate ansatz for the form fields [11,14]; in the case where the volume moduli $(\beta, \sigma)$ are fixed (or time-independent) the supergravity equations may be reduced to the form [14]

$$
\begin{gathered}
R_{\mu \nu}=0, \quad R_{\mu p}=0, \\
\hat{R}_{p q} \equiv R_{p q}-\frac{1}{n} R\left(X_{n}\right) g_{p q}\left(X_{n}\right)=0 .
\end{gathered}
$$

Here $(\mu, \nu)$ run from 1 to $(10-n)$. In particular, in the static case, $a \equiv a_{0}$, we define $\beta \equiv \beta(y), \sigma \equiv \sigma(y)$ [in the metric (3)] and take $n=6$. With $k \equiv y^{2}$, we find

$$
h=h_{0} \exp \left[c / y^{4}\right], \quad \beta=-\sqrt{6\left(1-2 c^{2} / y^{8}\right)} .
$$

In the large $y$ limit, $h(y \rightarrow \infty) \equiv h_{0}+L^{4} / y^{4}$ [11]. All our solutions, both for fixed and time-dependent volume moduli, satisfy the relation $\sigma=\beta-\frac{1}{2} \ln \left(3 f^{2} / 2\right)$, so we write down the result only for $\beta$. For the solution above only the region $y^{4}>\sqrt{2 c^{2}}$ is physical; the singularity at $y=0$ is due to the choice $k \equiv y^{2}$, not because of any specific ansatz for form fields. This is clear also from the deformed coni- fold solutions of [12]. To quantify this, suppose that $h \simeq$ $h_{0}$, without specifying $k(y)$. We find

$$
3\left(k^{\prime} / k\right)^{\prime}+k^{-1} e^{-2 \beta}=0 .
$$

Clearly, if $k \equiv k_{0} y^{2}$, then we get $\beta=-\ln \sqrt{6 k_{0}}$, while, if $k \equiv k_{0} \operatorname{sech}(y)$, then $\beta=-\ln \sqrt{3 k_{0}}+\frac{3}{2} \ln \cosh (y)$, which is regular everywhere.

The presence of external fluxes would modify the solution for warp and volume factors as in (9) for $a \equiv a_{0}$, or in a more complicated way for $a \equiv a(t)$. Since $R_{y y}=$ $\left(h^{4} k\right)^{-1 / 2} \frac{d}{d y}\left[h^{9 / 4} k^{3} \frac{d}{d y}\left(h^{-1 / 4} k^{-5 / 2}\right)\right]+\frac{9}{2} \frac{d}{d y}(\ln h) \frac{d}{d y}(\ln k)$ and $R_{t y}=-\frac{5}{2} \dot{\beta} \frac{d}{d y}[\ln (h k)]$, we find solutions only in the (large volume) limit where $h k=$ const (see below), or when the volume moduli are fixed. Moreover, $\mathcal{L} \rightarrow \mathcal{L}_{\mathrm{gr}}+\mathcal{L}_{\text {flux }}$, where $\mathcal{L}_{\text {flux }} \propto e^{-2 \phi-2 \sigma}\left(c_{1}^{2} e^{-4 \beta} h^{-2} k^{-3}+\frac{1}{2} e^{-8 \beta} h^{-3} k^{-5} K^{2}-\right.$ $\left.2 c_{1}^{2} e^{2 \sigma-4 \beta} F^{2} h^{-2} k^{-2}\right)$ with $K \equiv c_{0}+2 c_{1} F(y)$. Under our metric ansatz, equations $\hat{R}_{p q}=0$ (with $n=5$ ) are automatically satisfied. To see the effect of fluxes on the spatial sections of the cosmology, one can take $k \equiv y^{2}$ (and hence $\left.h=\sqrt{\lambda+\rho^{4} / y^{4}}\right)$. The explicit solution is

$$
a(t)=a_{0} e^{H t}, \quad H^{2} \equiv \sqrt{\frac{2}{3}} \frac{|f| \rho^{8} e^{-5 \beta}}{\left(\lambda y^{4}+\rho^{4}\right)^{5 / 2}},
$$

with an arbitrary (constant) $\beta$. In such a case $R_{p q}\left(X_{5}\right)$ is supported by five-form (and self-dual three-form) fluxes on $T^{1,1}$. The above solution is stable as long as the volume moduli are fixed. For slowly rolling moduli, any such de Sitter phase would be only metastable. This example demonstrates that it is indeed possible to maintain fixed volume modulus of the internal space while the spatial sections of the cosmology undergo a de Sitter expansion.

A constant warping. - In the large volume limit, when the backreaction of the fluxes on Einstein's equations can be ignored (since their contribution to the stress tensor is volume suppressed), the warp factor is minimized. This particular case may be related to the large $\tau$ limit of the resolved conifold metrics in [13], for which $h \rightarrow h(\tau) \equiv$ $h_{0}+h_{1} e^{-4 \tau / 3} ; k(\tau)$ and $m(\tau)$ also take their extremized values. The field equations reduce to equations of motion, and a constraint, for the variables $(\alpha, \beta, \sigma)$ that are the Euler-Lagrange equations of the effective Lagrangian $\mathcal{L}=\frac{3}{2} \dot{\alpha}^{2}+12 \dot{\beta}^{2}+\frac{3}{2} \dot{\sigma}^{2}+4 \dot{\alpha} \dot{\beta}+4 \dot{\beta} \dot{\sigma}+\frac{1}{2} \dot{\alpha} \dot{\sigma}-\frac{e^{-2 \phi-4 \beta}}{h_{0}} \times$ $\left(f^{2} e^{2 \sigma}-4 e^{2 \beta}\right)$. The explicit solution is

$$
\begin{gathered}
a=e^{\zeta u}(\cosh \chi u)^{-5 / 8}, \quad \alpha=c_{1} u+c_{0}, \\
\sigma=-\frac{1}{4} \ln \cosh \chi u+c_{2} u+c_{3}=\beta-\frac{1}{2} \ln \frac{3 f^{2}}{2}
\end{gathered}
$$

(in the gauge $\delta=3$ ), where $\zeta \equiv\left(c_{1}+7 c_{2}\right) / 6$ and

$$
h_{0}=\frac{64}{81} \frac{e^{-c_{0}-7 c_{3}}}{f^{6} \chi^{2}}, \quad \chi^{2} \equiv \frac{16}{15}\left(c_{1}^{2}+2 c_{1} c_{2}+7 c_{2}^{2}\right) .
$$

The four-dimensional cosmic time $t$ is defined by $d t=$ $\pm a^{3} d u$. It follows that this solution exhibits a period of 
accelerated expansion $(\dot{a}>0, \ddot{a}>0)$ in the 4D Einstein frame, provided that $2-3 \sqrt{2}<c_{1} / c_{2}<2+3 \sqrt{2}$. From a purely metric point of view, all the solutions with $|f|>0$ are nonsingular. The constants $c_{0}$ and $c_{3}$ may be set to zero using a shift-symmetry in $u$, or alternatively, can be absorbed into $g$ and $k$ so that each becomes unity even if they are assigned different values initially. The scalars $\beta$ and $\sigma$ can be stabilized by requiring that

$$
c_{1} / c_{2}=2 \text { or } c_{1} / c_{2}=-4 .
$$

The scale factor then evolves as $a \sim\left(e^{-c_{2} u}+e^{4 c_{2} u}\right)$ or as $a \sim\left(e^{-2 c_{2} u}+e^{3 c_{2} u}\right)$. In the first case the Universe still experiences a short period of accelerated acceleration before the volume scalars $\beta$ and $\sigma$ attain nearly fixed values, in which limit $V \propto e^{-\alpha}$. One takes $c_{2} u>0$, so that the physical three spaces expand faster, in the conventional manner. The radial modulus associated with $\mathbb{R}^{1}$ expands in the first case, providing a " $4+1+$ compact space" type background, while it shrinks in the second case, providing a " $3+1+$ compact space" type background. Thus the expansion of $3+1$ spacetime and contraction (or slow expansion) of the six extra dimensions can fundamentally be a natural phenomenon. Similar results exist in other versions of supergravity or string theory.

Consider ten-dimensional type IIA supergravity which already has a (positive) cosmological term [7]: $I_{\mathrm{gr}}=$ $\frac{1}{8 \pi G_{10}} \int \sqrt{-g_{10}}(R-2 \Lambda)$. In the case $h(y) \rightarrow h_{0}$, the 10D Einstein equations are solved by

$$
\begin{gathered}
k=\frac{1}{4 \nu^{2}}\left[k_{1} \sin (\nu y)-k_{2} \cos (\nu y)\right]^{2}, \quad \nu \equiv \sqrt{\sqrt{h_{0}} \Lambda / 20}, \\
a=a_{0} e^{H t}, \quad H \equiv e^{-2 \beta-\sigma / 2} \sqrt{\Lambda /\left(12 \sqrt{h_{0}}\right)}, \\
\beta=\frac{1}{2} \ln \frac{2}{3\left(k_{1}^{2}+k_{2}^{2}\right)}=\sigma+\frac{1}{2} \ln \frac{3 f^{2}}{2},
\end{gathered}
$$

where $k_{1}, k_{2}$ are arbitrary constants. This solution clearly supports an accelerated expansion for $\Lambda>0$. It would be interesting to see a generalization of this result in the case $h \equiv h(y)$.

We conclude the Letter with a short summary of the results. It has been a difficult problem to construct accelerating cosmologies from toroidal or spherical compactification of string or supergravity theory with stabilized or slowly rolling volume moduli. In this Letter we have shown that it is possible to construct an effective fourdimensional cosmology undergoing one or more periods of accelerated expansion in the general setting of 10D supergravity compactified on a warped $6 \mathrm{D}$ conifold, with or without external fluxes. Allowing time dependence in the warped conifold solutions is an excellent route for studying aspects of de Sitter Universe via string compactifications. We considered explicit cosmologies that arise in models of gravity which correspond to the dimensional reduction to four dimensions of 10D supergravity. It is remarkable that a model with so many attractive features can arise from a simple compactification of type IIB (as well as type IIA) supergravity on a warped conifold. Further generalizations of the solutions discovered in this Letter are also possible, including the case where $g=g(y)$. Finally, we note that for slowly rolling moduli, the effective potential, $V(\phi)$, can vary slowly with time, while for fixed volume moduli, it acts purely as a cosmological term [cf. (11)]; thus the model could satisfy the solar system test and other constraints from cosmology.

I wish to thank Igor Klebanov for constructive remarks and recommendations on the draft, and Chiang-Mei Chen, Chris Herzog, Pei-Ming Ho, David Wiltshire for helpful discussions. This research is supported by the Foundation for Research, Science and Technology (NZ) under Research Grant No. E5229.

*Email address: Ishwaree.Neupane @ cern.ch

[1] S. Perlmutter et al., Astrophys. J. 517, 565 (1999); A. G. Riess et al., Astrophys. J. 607, 665 (2004).

[2] D. N. Spergel et al., Astrophys. J. Suppl. Ser. 148, 175 (2003).

[3] S. Kachru, R. Kallosh, A. Linde, and S. P. Trivedi, Phys. Rev. D 68, 046005 (2003).

[4] P. K. Townsend and M. N. R. Wohlfarth, Phys. Rev. Lett. 91, 061302 (2003); [see also, N. Ohta, Phys. Rev. Lett. 91, 061303 (2003)].

[5] C.-M. Chen, P.-M. Ho, I. P. Neupane, N. Ohta, and J.E. Wang, J. High Energy Phys. 10 (2003) 058 (see also references therein).

[6] G.W. Gibbons, in Supersymmetry, Supergravity and Related Topics, edited by F. del Aguila, J.A. de Azcarraga, and L.E. Ibanez (World Scientific, Singapore, 1985), pp. 123-146.

[7] J. M. Maldacena and C. Nunez, Int. J. Mod. Phys. A 16, 822 (2001).

[8] Y. M. Cho and I. P. Neupane, Int. J. Mod. Phys. A 18, 2703 (2003).

[9] S. B. Giddings, S. Kachru, and J. Polchinski, Phys. Rev. D 66, 106006 (2002).

[10] I. P. Neupane and D. L. Wiltshire, Phys. Lett. B 619, 201 (2005); Phys. Rev. D 72, 083509 (2005).

[11] I. R. Klebanov and A. A. Tseytlin, Nucl. Phys. B578, 123 (2000).

[12] I. R. Klebanov and M. J. Strassler, J. High Energy Phys. 08 (2000) 052; C. P. Herzog, I. R. Klebanov, and P. Ouyang, hep-th/0205100.

[13] L. A. Pando Zayas and A. A. Tseytlin, J. High Energy Phys. 11 (2000) 028.

[14] H. Kodama and K. Uzawa, J. High Energy Phys. 07 (2005) 061; 03 (2006) 053.

[15] A. Buchel, Phys. Rev. D 65, 125015 (2002). 